\title{
Biopsy for advanced hepatocellular carcinoma: results of a multicentre UK audit
}

\begin{abstract}
Alexa Childs ${ }^{1}$, Nekisa Zakeri ${ }^{1}$, Yuk Ting $\mathrm{Ma}^{2}$, Joanne O'Rourke ${ }^{2}$, Paul Ross ${ }^{3}$, Essam Hashem ${ }^{3}$, Richard A. Hubner ${ }^{4}$, Kimberley Hockenhull ${ }^{4}$, Chinenye Iwuji ${ }^{5}$, Sam Khan ${ }^{5}$, Daniel H. Palmer ${ }^{6}$, Joanna Connor ${ }^{6}$, Daniel Swinson ${ }^{7}$, Suzanne Darby ${ }^{8}$, Chiara Braconi ${ }^{9}$, Tom Roques ${ }^{10}$, Dominic Yu ${ }^{11}$, Tu Vinh Luong ${ }^{12}$ and Tim Meyer (D) $^{1,13 凶}$
\end{abstract}

(c) The Author(s) 2021

BACKGROUND: Advanced hepatocellular carcinoma (HCC) is commonly diagnosed using non-invasive radiological criteria (NIRC) defined by the European Association for the Study of the Liver or the American Association for the Study of Liver Diseases. In 2017, The National Institute for Clinical Excellence mandated histological confirmation of disease to authorise the use of sorafenib in the UK.

METHODS: This was a prospective multicentre audit in which patients suitable for sorafenib were identified at multidisciplinary meetings. The primary analysis cohort (PAC) was defined by the presence of Child-Pugh class A liver disease and performance status 0-2. Clinical, radiological and histological data were reported locally and collected on a standardised case report form. RESULTS: Eleven centres reported 418 cases, of which 361 comprised the PAC. Overall, 76\% had chronic liver disease and $66 \%$ were cirrhotic. The diagnostic imaging was computed tomography in $71 \%$, magnetic resonance imaging in $27 \%$ and $2 \%$ had both. Preexisting histology was available in 45 patients and 270 underwent a new biopsy, which confirmed HCC in 93.4\%. Alternative histological diagnoses included cholangiocarcinoma (CC) and combined HCC-CC. In cirrhotic patients, NIRC criteria had a sensitivity of $65.4 \%$ and a positive predictive value of $91.4 \%$ to detect HCC. Two patients $(0.7 \%)$ experienced mild post-biopsy bleeding. CONCLUSION: The diagnostic biopsy is safe and feasible for most patients eligible for systemic therapy

British Journal of Cancer (2021) 125:1350-1355; https://doi.org/10.1038/s41416-021-01535-2

\section{BACKGROUND}

The non-invasive radiological criteria (NIRC) for the diagnosis of hepatocellular carcinoma (HCC) were first defined by the European Association for the Study of the Liver (EASL) in 2001, but have been refined over time [1]. In the current version of the guidelines, a diagnosis of HCC can be made when a nodule $\geq 1 \mathrm{~cm}$ occurring in a cirrhotic liver displays both arterial phase hyperenhancement and washout on the portal venous or delayed phase using multiphasic computed tomography (CT) or dynamic contrast-enhanced magnetic resonance imaging (MRI) [2]. The American Association for the Study of Liver Diseases (AASLD) also define the diagnosis of HCC by these criteria, but additionally consider threshold growth as described by Liver Imaging Reporting and Data System (LI-RADS) [3]. The majority of studies investigating the diagnostic accuracy of NIRC are retrospective and the focus has been on the detection of early disease $[4,5]$. Despite this, the diagnostic criteria have been extended to advanced disease with the consequence that patients with HCC usually embark on systemic therapy without a histological diagnosis. In this respect, HCC is unique among solid tumours and, in the era of precision medicine, HCC research has been disadvantaged by the lack of archival tissue from patients with advanced disease. Arguments advanced against biopsy include the risk of tumour seeding and bleeding. However, there remain very limited data on the diagnostic accuracy of imaging and the complication rate associated with biopsy in patients with advanced disease.

In November 2017, NHS England mandated histological confirmation of HCC prior to the initiation of sorafenib. This was based on the fact that pathological diagnosis was required for the SHARP trial that led to the registration of sorafenib [6]. The requirement for biopsy was only waived in exceptional circumstances where a biopsy was deemed to be too high risk or technically not feasible in the opinion of the specialist HCC

\footnotetext{
${ }^{1}$ Department of Oncology, Royal Free London NHS Foundation Trust, London, UK. ${ }^{2}$ University Hospitals Birmingham NHS Foundation Trust, Birmingham, UK. ${ }^{3}$ King's College

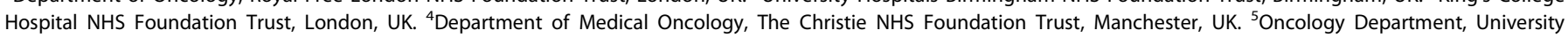

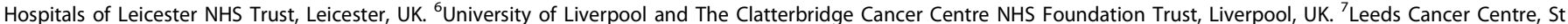

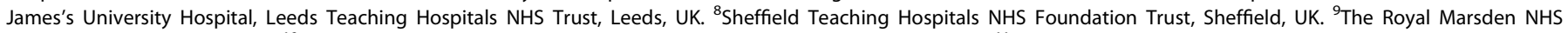

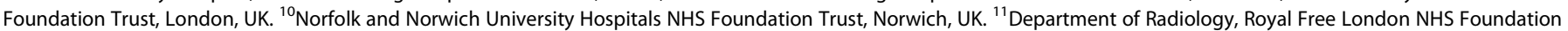
Trust, London, UK. ${ }^{12}$ Department of Cellular Pathology, Royal Free London NHS Foundation Trust, London, UK. ${ }^{13}$ UCL Cancer Institute, University College London, London, UK. 凶email: t.meyer@ucl.ac.uk
}

Received: 11 June 2021 Revised: 4 August 2021 Accepted: 20 August 2021

Published online: 15 September 2021 


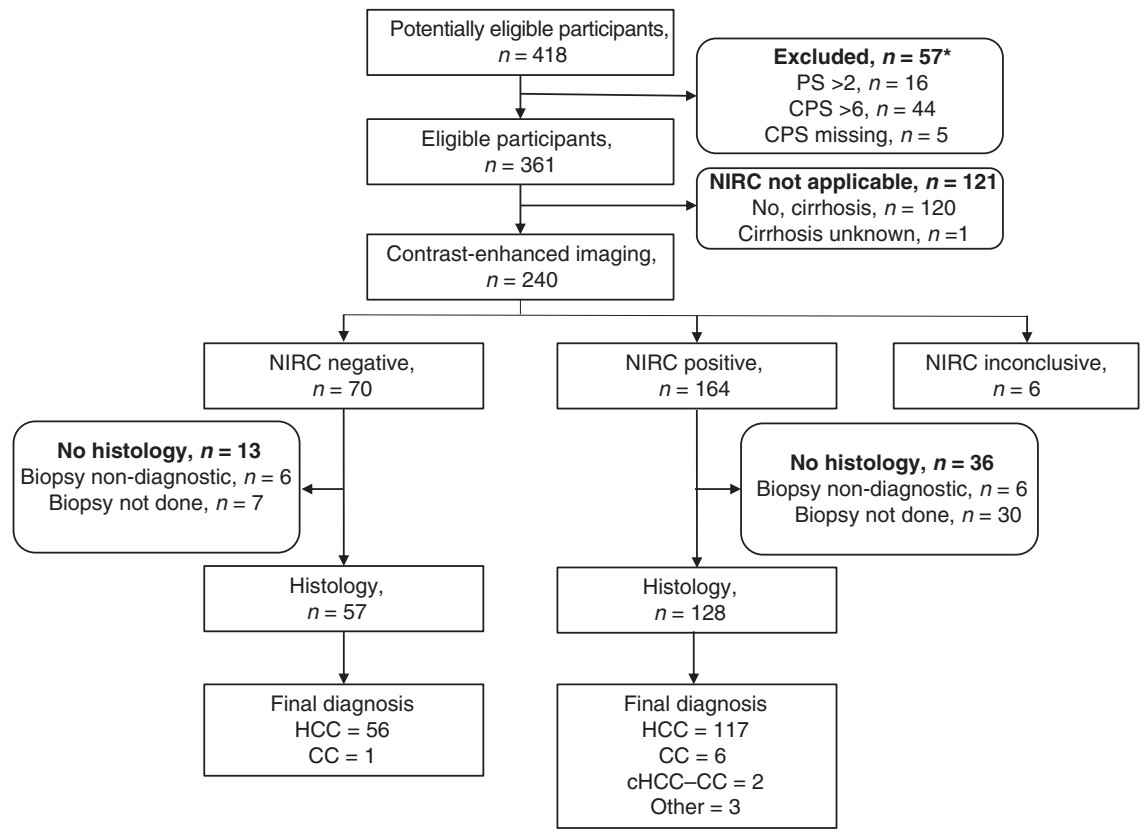

Fig. 1 STARD flow diagram. *Eight patients had more than one reason. PS performance status, CPS Child-Pugh score.

multidisciplinary team (MDT) meeting in a patient who otherwise met the non-invasive diagnostic criteria of HCC. The requirement for biopsy permitted the conduct of a multicentre, prospective audit to establish the safety and outcome of biopsy in patients with suspected advanced HCC.

\section{METHODS}

Eleven UK centres providing specialist care for patients with liver cancer agreed to participate and were provided with a standardised report form to enable the prospective collection of anonymised data. Patients were included if they were deemed suitable for systemic therapy for advanced $\mathrm{HCC}$ at a dedicated MDT meeting and subsequently assessed in the local clinic. Patient characteristics including age, sex, disease aetiology, presence of cirrhosis, alpha-fetoprotein (AFP) and Child-Pugh score were recorded. Contrast-enhanced CT and/or MRI was performed, and centres were required to report the presence of arterial enhancement, washout in the portal venous phase and radiological evidence of chronic liver disease as evidenced by liver contour, varices and splenomegaly. In addition, the number of tumours, size of largest tumour, presence of macrovascular invasion and extrahepatic spread were also documented. In the absence of previous histology or perceived high risk of biopsy, percutaneous tumour biopsy was performed and histology was reported locally. The primary analysis cohort (PAC) comprised all those who met the criteria for sorafenib as defined by National Institute for Clinical Excellence (NICE) guidelines, namely Child-Pugh class A liver disease and performance status (PS) $\leq 2$. However, all patients were included in the safety analysis. This study was classified as a national audit and clinical evaluation by the Caldecott Guardian, the primary aim of which was to evaluate the feasibility, safety and outcome of diagnostic liver biopsy. As such, individual patient consent was not required. The study was reported according to Standards for Reporting Diagnostic accuracy studies (STARD) guidelines.

\section{RESULTS}

\section{Patient characteristics}

Overall, 11 UK hospitals contributed to this audit and data were submitted on 418 patients identified between January 2018 and August 2020. Fifty-seven patients who were not confirmed to be Child-Pugh class A and PS $\leq 2$ were excluded, leaving a total of 361 for the PAC (see STARD flow diagram in Fig. 1 and Table 1). The median age was 68 , the majority were male and $83 \%$ had a PS of 0 or 1 . Chronic liver disease was present in $76 \%$ with HCV, alcohol and NASH/NAFLD accounting for the majority, each being present in broadly equivalent proportion. Sixty-six per cent of patients were cirrhotic. More than one risk factor for chronic liver disease was present in $12 \%$. At least one previous locoregional or surgical intervention had been delivered in $43 \%$, with the most common being arterial embolic treatment, and $11 \%$ had received at least two transarterial embolisation/transarterial chemoembolisation procedures. AFP was $\geq 400 \mathrm{ng} / \mathrm{mL}$ in $37 \%$.

\section{Radiological and histological evaluation}

Imaging characteristics are shown in Table 2. CT was the diagnostic modality in $71 \%, \mathrm{MRI}$ in $27 \%$ and seven patients (2\%) had both. The median size of the largest tumour was $6 \mathrm{~cm}$ and three patients with prior resection had extrahepatic disease only with no evidence of intrahepatic disease. The majority had three lesions or less, $42 \%$ had an extrahepatic disease and $34 \%$ had a macrovascular invasion. Of the 361 in the PAC, 120 did not have cirrhosis and 1 was unknown, leaving 240 that were evaluable by NIRC (Fig. 1). The NIRC was fulfilled for 164 of the 240 patients. The remaining 76 patients did not meet the criteria; 43 did not demonstrate arterialisation, 64 did not demonstrate portal venous washout and in six cases the imaging was not evaluable due to missing sequences. As shown in Table 3, prior histological diagnosis of HCC was available for 45 patients and 270 underwent a new tumour biopsy, of which 237 confirmed HCC, and thus giving a total of 282 patients with HCC. Although HCC accounted for $93 \%$ of the histological diagnoses, a variety of other diagnoses were made, including cholangiocarcinoma (CC), combined HCC (cHCC)-CC, benign lesions and 'others', which included neuroendocrine and breast carcinoma (Fig. 1 and Table 3). Biopsy was nondiagnostic in 13 patients and was not performed in an additional 45 patients due to poor accessibility, low platelets, clotting derangement or ascites (Table 3). In $25 \%$ the reason for not performing a biopsy was not given. The NIRC are only applicable to those with cirrhosis, and in order to calculate the specificity and positive predictive value (PPV) of the NIRC using histology as the gold standard, those with cirrhosis and those meeting NIRC were separated as shown in Table 4. CT and MRI were analysed both together and separately. Overall, the sensitivity for NIRC to correctly identify HCC was $65.4 \%$ and was slightly higher for MRI compared to CT at $68.9 \%$ and $64.6 \%$, respectively. The overall PPV 
Table 1. Patient characteristics.

\begin{tabular}{|c|c|c|}
\hline Characteristic & $n=361$ & $\%$ \\
\hline Median age (range) & $68(21-87)$ & \\
\hline \multicolumn{3}{|l|}{ Gender } \\
\hline Male & 296 & 82 \\
\hline Female & 65 & 18 \\
\hline \multicolumn{3}{|l|}{ Performance status } \\
\hline 0 & 77 & 21 \\
\hline 1 & 225 & 62 \\
\hline 2 & 60 & 17 \\
\hline \multicolumn{3}{|l|}{ Child-Pugh score } \\
\hline 5 & 270 & 75 \\
\hline 6 & 91 & 25 \\
\hline \multicolumn{3}{|l|}{ Chronic liver disease } \\
\hline Yes & 273 & 76 \\
\hline No & 88 & 24 \\
\hline \multicolumn{3}{|c|}{ Aetiology of chronic liver disease } \\
\hline HCV & 71 & 20 \\
\hline HBV & 33 & 9 \\
\hline Alcohol & 88 & 24 \\
\hline NASH/NAFLD & 72 & 20 \\
\hline Other & 36 & 10 \\
\hline Unknown & 18 & 5 \\
\hline \multicolumn{3}{|l|}{ Cirrhosis } \\
\hline Yes & 240 & 66 \\
\hline No & 120 & 33 \\
\hline Unknown & 1 & 0.3 \\
\hline \multicolumn{3}{|l|}{ Prior treatment } \\
\hline None & 207 & 57 \\
\hline TACE/TAE & 122 & 34 \\
\hline Surgery & 37 & 10 \\
\hline Ablation, & 20 & 6 \\
\hline \multicolumn{3}{|l|}{ Baseline AFP } \\
\hline$<400 \mathrm{ng} / \mathrm{mL}$ & 222 & 61 \\
\hline$\geq 400 \mathrm{ng} / \mathrm{mL}$ & 133 & 37 \\
\hline
\end{tabular}

HCV hepatitis C virus, HBV hepatitis B virus, NASH non-alcoholic steatohepatitis, NAFLD non-alcoholic fatty liver disease, TAE transarterial embolisation, TACE transarterial chemoembolisation, AFP alphafetoprotein.

was $91.4 \%$ and again was slightly better for MRI at $93.9 \%$ compared with $90.3 \%$ for CT. The inclusion of 45 patients with prior histology would tend to overestimate the sensitivity and PPV, so the data were also analysed for those who had fresh biopsy only. Only 11 of 45 with prior histology had imaging that met NIRC, and when these patients were excluded, the overall sensitivity reduced to $63.1 \%$ and the PPV was $90.6 \%$. The 120 patients who were not cirrhotic, by definition, did not meet the NIRC and so would be recommended to have a biopsy as per international guidelines. Of these, 55 had lesions demonstrating arterialisation and washout. Overall, 102 (85\%) had histological confirmation of HCC, and of the 55 with characteristic enhancement patterns, 46 (82\%) had histological confirmation of HCC, 3 had CC, 1 was benign, 1 was non-diagnostic and 4 could not have a biopsy for technical reasons.
Table 2. Radiological findings.

\begin{tabular}{|llr|}
\hline Radiological findings & $\boldsymbol{n = 3 6 1}$ & $\%$ \\
\hline $\begin{array}{l}\text { Median size of the largest liver lesion (range) }(\mathrm{cm}) \\
\text { No. of lesions }\end{array}$ & $6(0-19)$ & \\
\hline $0-3$ & 185 & 51.2 \\
\hline$>3$ & 164 & 45.4 \\
\hline NK & 12 & 3.3 \\
\hline Extrahepatic disease & 151 & 41.8 \\
\hline Macrovascular Invasion & 121 & 33.5 \\
\hline Tumour arterialisation & 269 & 74.5 \\
\hline Portal venous washout & 230 & 63.7 \\
\hline Radiological evidence of chronic liver disease & 230 & 63.7 \\
\hline Met NIRC for HCC & 164 & 45.4
\end{tabular}

NK not known.

Table 3. Results of biopsy.

\begin{tabular}{|c|c|c|}
\hline & $n=361$ & $\%$ \\
\hline \multicolumn{3}{|l|}{ Biopsy } \\
\hline Yes & 270 & \\
\hline No & 91 & \\
\hline \multicolumn{3}{|c|}{ Reason for not performing biopsy $(n=91)$} \\
\hline Prior histology of HCC & 45 & 49.5 \\
\hline Not accessible & 9 & 9.9 \\
\hline Low platelets & 6 & 6.6 \\
\hline Clotting & 4 & 4.4 \\
\hline Ascites & 1 & 1.1 \\
\hline Other & 26 & 28.6 \\
\hline \multicolumn{3}{|l|}{ Histology $(n=302)$} \\
\hline $\mathrm{HCC}$ & 282 & 93.4 \\
\hline HCC (new biopsy) & 237 & 78.5 \\
\hline HCC (prior histology) & 45 & 14.9 \\
\hline Cholangiocarcinoma (CC) & 11 & 3.6 \\
\hline Combined HCC-CC & 2 & 0.7 \\
\hline Benign & 1 & 0.3 \\
\hline Other & 6 & 2.0 \\
\hline Non-diagnostic & 13 & \\
\hline
\end{tabular}

Adverse events. Among the PAC comprising 361 patients, in which 270 new biopsies were performed, there were two adverse events. In both cases, mild bleeding was reported, but there was no haemodynamic compromise or fall in haemoglobin, and no intervention was required. Among the entire submitted cohort of 418 , which included those not eligible for systemic therapy due to Child-Pugh score or PS, there were two additional reports of bleeding among 317 biopsy procedures. In one case, the patient developed a high white count and CRP post biopsy and was found to have $100 \mathrm{~mL}$ free fluid on $\mathrm{CT}$, which was compatible with postbiopsy bleeding. No intervention was required, and the patient recovered spontaneously. In the second case, the patient died from post-biopsy haemorrhage. This patient had Child-Pugh score of 7 and an international normalised ratio of 1.34. In summary, for those eligible for sorafenib, the rate of mild bleeding was $0.7 \%$ and there were no serious sequelae, but beyond these criteria, the risk of serious complications increases. There were no reports of tumour seeding. 
Table 4. Concordance of radiology and histology.

\begin{tabular}{|c|c|c|c|c|c|c|c|c|c|}
\hline & Tota & & & Cirrh & & & NIRC & & \\
\hline & $A I^{\mathbf{a}}$ & CT & MRI & $\mathbf{A l l}^{\mathbf{b}}$ & CT & MRI & AlI ${ }^{c}$ & Ст & MRI \\
\hline & 361 & 257 & 97 & 240 & 174 & 62 & 164 & 121 & 41 \\
\hline $\mathrm{HCC}$ & 282 & 201 & 74 & 179 & 130 & 45 & 117 & 84 & 31 \\
\hline $\mathrm{CC}$ & 11 & 8 & 3 & 7 & 6 & 1 & 6 & 5 & 1 \\
\hline $\mathrm{HCC}-\mathrm{CC}$ & 2 & 1 & 1 & 2 & 1 & 1 & 2 & 1 & 1 \\
\hline Other & 6 & 6 & 0 & 3 & 3 & 0 & 3 & 3 & 0 \\
\hline Total non-HCC & 20 & 16 & 4 & 12 & 10 & 2 & 11 & 9 & 2 \\
\hline Non-diagnostic & 13 & 9 & 4 & 12 & 9 & 3 & 6 & 6 & 0 \\
\hline No histology & 46 & 30 & 13 & 37 & 25 & 12 & 30 & 22 & 8 \\
\hline
\end{tabular}

${ }^{\mathrm{a} S}$ Seven had CT and MRI.

${ }^{\mathrm{b}}$ Four had CT and MRI.

'Two had CT and MRI.

Treatment. For the 282 patients with histologically confirmed HCC, $88 \%$ went on to receive systemic therapy; 223 (79\%) received sorafenib, $26(9 \%)$ received a clinical trial or alternative systemic therapy, 8 (3\%) had best supportive care and 7 (2\%) declined therapy. For $18(6 \%)$, the patient had transferred to another hospital or treatment was not documented.

\section{DISCUSSION}

To our knowledge, this is the largest analysis of NIRC applied to advanced HCC, in which histology has been used as the gold standard. The patient population evaluated here is typical of that offered systemic therapy in routine practice and clinical trials. Given the criteria for sorafenib eligibility defined by NICE, we confined our central analysis to those with Child-Pugh class $A$ and PS 0-2 (the PAC). Indeed, there is good evidence to support restricting treatment to this subgroup with multiple studies demonstrating the very limited benefit of sorafenib to those outside these criteria [7-9]. Within the PAC population, $76 \%$ had at least one cause of chronic liver disease, but only $66 \%$ were cirrhotic, and only $45 \%$ met NIRC. Hence, $65 \%$ would have required confirmatory biopsy to make a confident diagnosis. The overall sensitivity and PPV of NIRC in our population were $65.4 \%$ and $91.4 \%$, respectively, and this is comparable with previous studies on early disease. A meta-analysis, including 242 studies in which the radiological criteria were not uniformly defined, reported a per-lesion sensitivity and PPV of $73.6 \%$ and $85.8 \%$, respectively, for $\mathrm{CT}$ and $77.5 \%$ and $83.6 \%$, respectively, for gadolinium-enhanced MRI [10]. Both sensitivity and PPV were lower in 'explant-only' studies where pathological confirmation was available. A more recent meta-analysis evaluating LI-RADS reported a sensitivity of $66 \%$ for LI-RADS $\geq 5$ [5]. In early disease, the criteria are generally used to distinguish between benign and malignant disease, whereas in advanced disease, the criteria are being used to differentiate HCC from another malignancy. Whilst PPV reported in our study and others may be acceptable for earlystage disease, it is far from satisfactory in the setting of advanced disease where reliance on NIRC alone will result in around 10\% receiving an incorrect diagnosis. In our study, alternative diagnoses including $\mathrm{CC}$, CHCC-CC and a variety of other tumours were confirmed by biopsy, implying that reliance on imaging alone would have resulted in these patients receiving potentially inappropriate therapy. CC also occurs in the context of chronic liver disease and previous studies have reported similar enhancement patterns in both intrahepatic CC and HCC in cirrhotic patients [11]. Our study serves to confirm these findings. Similarly, the overlap between imaging characteristics of CHCC-CC and HCC has recently been highlighted and biopsy has been recommended to improve diagnostic accuracy [12-14].

The most recent version of the EASL guidelines defines the criteria for radiological diagnosis of HCC, but states that 'upfront liver biopsy and blood sampling is recommended for clinical and diagnostic trials' [2]. However, histological diagnosis has only been mandatory for a relatively small number of phase III trials [6, 15-18] and the most recently approved agents have been evaluated in clinical trials for which pathological confirmation was not required [19-22]. Given our findings, it is of particular concern that registrational trials, which have not mandated biopsy, may have recruited up to $10 \%$ of patients with a diagnosis other than HCC.

An additional factor limiting the adoption of routine biopsy in $\mathrm{HCC}$ is the risk of complications, which may include pain, bleeding, tumour seeding and death. Mild bleeding is reported in around $3-4 \%$ cases of liver biopsy and severe bleeding requiring transfusion in $0.5 \%$ [23]. In a more recent study that included $>1100$ biopsies, there were four bleeding events accounting for an incidence $<0.4 \%$ [18]. Mortality is generally considered to be around 1 in 10,000 [23]. The risk of biopsy is considered to be higher in those with underlying cirrhosis, but in our series, the rate of mild bleeding was only $0.7 \%$. One patient with Child-Pugh class $B$ had severe bleeding from which he died, underlining the increased risk in patients with liver dysfunction. Patients with Child-Pugh class B disease were not included in the seminal SHARP trial and are not eligible for systemic therapy according to NICE guidelines. In the field of practice studies, the outcome for patients with Child-Pugh class B disease treated with sorafenib was poor with a median survival of 5.2 months [8], and recent data for nivolumab in this patient population confirms the poor outlook [24]. Needle track seeding was originally reported at 2.7 or $0.9 \%$ per year [25], but subsequent large studies have reported lower rates ranging from 0.14 to $0.76 \%$ [26-28]. In the context of advanced disease, the risk of seeding is of less concern and in practice was not reported at all in our series.

The lack of tissue from advanced HCC treated in clinical trials and routine practice has limited the capacity for the widespread genomic analysis and biomarker development that has characterised progress in other cancers. Consequently, the majority of publications exploring the genomics of HCC have been conducted on early-stage, resected tumours, many of which occur in noncirrhotic livers and therefore may not be representative of tumour biology in advanced disease [29-33]. Furthermore, the molecular divergence has been demonstrated in metastatic tumours [34] and the extent of molecular evolution from early- to late-stage 
disease has not been well defined. The evidence presented here provides a clear rationale to undertake biopsy in patients with suspected advanced HCC and this will help to accelerate our understanding of cancer biology in advanced HCC.

Despite being a large multicentre prospective study, we acknowledge some limitations. First, although all cases were reviewed by a local specialist hepatobiliary MDT, there was no central review of radiology or histology. It is therefore not possible to differentiate between a true failure of the NIRC to correctly diagnose HCC or a local misinterpretation of the imaging. However, the 11 false-positive cases were evenly distributed across four high-volume centres, suggesting that the findings reflect real-life clinical practice rather than attributable to systematic issues with one centre. Second, it is likely that the false-negative rate may have been underestimated if these patients did not undergo biopsy, but this would imply that the true sensitivity is even lower than we report. Finally, only a minority of patients had both CT and MRI, which could have reduced the sensitivity in our study. However, the sensitivity that we report is in line with other studies and the lack of dual imaging does not undermine the key finding that up to $10 \%$ of those who met the NIRC did not have HCC.

\section{CONCLUSION}

We demonstrate that the majority of patients with advanced HCC do not present with diagnostic imaging, and in those who do, up to one in ten will have a diagnosis other than HCC. In patients with a preserved liver function who are eligible for systemic therapy, tumour biopsy proved to be safe and provided a histological diagnosis of HCC or other cancer in $96 \%$ of cases. In the era of precision medicine, we recommend that biopsy should be routinely performed in patients with suspected advanced HCC who are potentially eligible for systemic therapy. The adoption of EASL's recommendations to incorporate tissue sampling into clinical trials is also strongly endorsed.

\section{REFERENCES}

1. Bruix J, Sherman M, Llovet JM, Beaugrand M, Lencioni R, Burroughs AK. et al. Clinical management of hepatocellular carcinoma. Conclusions of the Barcelona2000 EASL conference. European Association for the Study of the Liver. J Hepatol. 2001;35:421-30.

2. European Association for the Study of the Liver. EASL Clinical Practice Guidelines: management of hepatocellular carcinoma. J Hepatol. 2018;69:182-236.

3. Marrero JA, Kulik LM, Sirlin CB, Zhu AX, Finn RS, Abecassis MM, et al. Diagnosis, staging, and management of hepatocellular carcinoma: 2018 Practice Guidance by the American Association for the Study of Liver Diseases. Hepatology. 2018;68:723-50.

4. Liu X, Jiang H, Chen J, Zhou $Y$, Huang Z, Song B. Gadoxetic acid disodiumenhanced magnetic resonance imaging outperformed multidetector computed tomography in diagnosing small hepatocellular carcinoma: a meta-analysis. Liver Transplant. 2017;23:1505-18.

5. Liang Y, Xu F, Guo Y, Lai L, Jiang X, Wei X, et al. Diagnostic performance of LIRADS for MRI and CT detection of HCC: a systematic review and diagnostic metaanalysis. Eur J Radiol. 2021;134:109404.

6. Llovet JM, Ricci S, Mazzaferro V, Hilgard P, Gane E, Blanc JF, et al. Sorafenib in advanced hepatocellular carcinoma. N. Engl J Med. 2008;359:378-90.

7. King J, Palmer DH, Johnson $P$, Ross $P$, Hubner RA, Sumpter $K$, et al. Sorafenib for the treatment of advanced hepatocellular cancer - a UK audit. Clin Oncol (R Coll Radiol). 2017;29:256-62.

8. Marrero JA, Kudo M, Venook AP, Ye SL, Bronowicki JP, Chen XP, et al. Observational registry of sorafenib use in clinical practice across Child-Pugh subgroups: the GIDEON study. J Hepatol. 2016;65:1140-7.

9. Pressiani T, Boni C, Rimassa L, Labianca R, Fagiuoli S, Salvagni S, et al. Sorafenib in patients with Child-Pugh class $A$ and $B$ advanced hepatocellular carcinoma: a prospective feasibility analysis. Ann Oncol. 2013;24:406-11.

10. Hanna RF, Miloushev VZ, Tang A, Finklestone LA, Brejt SZ, Sandhu RS, et al. Comparative 13-year meta-analysis of the sensitivity and positive predictive value of ultrasound, CT, and MRI for detecting hepatocellular carcinoma. Abdom Radiol. 2016;41:71-90.
11. Huang $B, W u L$, Lu XY, Xu F, Liu CF, Shen WF, et al. Small intrahepatic cholangiocarcinoma and hepatocellular carcinoma in cirrhotic livers may share similar enhancement patterns at multiphase dynamic MR imaging. Radiology. 2016;281:150-7.

12. Beaufrere A, Calderaro J, Paradis V. Combined hepatocellular-cholangiocarcinoma: an update. J Hepatol. 2021;74:1212-24.

13. Gigante $E$, Ronot $M$, Bertin C, Ciolina M, Bouattour M, Dondero F, et al. Combining imaging and tumour biopsy improves the diagnosis of combined hepatocellularcholangiocarcinoma. Liver Int. 2019;39:2386-96.

14. Li R, Yang D, Tang CL, Cai P, Ma KS, Ding SY, et al. Combined hepatocellular carcinoma and cholangiocarcinoma (biphenotypic) tumors: clinical characteristics, imaging features of contrast-enhanced ultrasound and computed tomography. BMC Cancer. 2016;16:158.

15. Johnson PJ, Qin S, Park JW, Poon RT, Raoul JL, Philip PA, et al. Brivanib versus sorafenib as first-line therapy in patients with unresectable, advanced hepatocellular carcinoma: results from the randomized phase III BRISK-FL study. J Clin Oncol. 2013;31:3517-24.

16. Llovet JM, Decaens T, Raoul JL, Boucher E, Kudo M, Chang C, et al. Brivanib in patients with advanced hepatocellular carcinoma who were intolerant to sorafenib or for whom sorafenib failed: results from the randomized phase III BRISKPS study. J Clin Oncol. 2013;31:3509-16.

17. Abou-Alfa GK, Meyer T, Cheng AL, El-Khoueiry AB, Rimassa L, Ryoo BY, et al. Cabozantinib in patients with advanced and progressing hepatocellular carcinoma. N Engl J Med. 2018;379:54-63.

18. Rimassa L, Assenat E, Peck-Radosavljevic M, Pracht M, Zagonel V, Mathurin P, et al. Tivantinib for second-line treatment of MET-high, advanced hepatocellular carcinoma (METIV-HCC): a final analysis of a phase 3, randomised, placebocontrolled study. Lancet Oncol. 2018;19:682-93.

19. Finn RS, Qin S, Ikeda M, Galle PR, Ducreux M, Kim TY, et al. Atezolizumab plus bevacizumab in unresectable hepatocellular carcinoma. $\mathrm{N}$ Engl J Med. 2020;382:1894-905.

20. Bruix J, Qin S, Merle P, Granito A, Huang YH, Bodoky G, et al. Regorafenib for patients with hepatocellular carcinoma who progressed on sorafenib treatment (RESORCE): a randomised, double-blind, placebo-controlled, phase 3 trial. Lancet. 2017;389:56-66.

21. Kudo M, Finn RS, Qin S, Han KH, Ikeda K, Piscaglia F, et al. Lenvatinib versus sorafenib in first-line treatment of patients with unresectable hepatocellular carcinoma: a randomised phase 3 non-inferiority trial. Lancet. 2018;391:1163-73.

22. Zhu AX, Kang YK, Yen CJ, Finn RS, Galle PR, Llovet JM, et al. Ramucirumab after sorafenib in patients with advanced hepatocellular carcinoma and increased alpha-fetoprotein concentrations (REACH-2): a randomised, double-blind, placebo-controlled, phase 3 trial. Lancet Oncol. 2019;20:282-96.

23. Rockey DC, Caldwell SH, Goodman ZD, Nelson RC, Smith AD. American Association for the Study of Liver Diseases. Liver biopsy. Hepatology. 2009;49:1017-44.

24. Kudo M, Matilla A, Santoro A, Melero I, Gracian AC, Acosta-Rivera M, et al. CheckMate 040 cohort 5: a phase I/II study of nivolumab in patients with advanced hepatocellular carcinoma and Child-Pugh B cirrhosis. J Hepatol. 2021;75:600-9.

25. Silva MA, Hegab B, Hyde C, Guo B, Buckels JA, Mirza DF. Needle track seeding following biopsy of liver lesions in the diagnosis of hepatocellular cancer: $a$ systematic review and meta-analysis. Gut. 2008;57:1592-6.

26. Ahn DW, Shim JH, Yoon JH, Kim CY, Lee HS, Kim YT, et al. Treatment and clinical outcome of needle-track seeding from hepatocellular carcinoma. Korean J Hepatol. 2011;17:106-12.

27. Chang S, Kim SH, Lim HK, Lee WJ, Choi D, Lim JH. Needle tract implantation after sonographically guided percutaneous biopsy of hepatocellular carcinoma: evaluation of doubling time, frequency, and features on CT. Am J Roentgenol. 2005;185:400-5.

28. Szpakowski JL, Drasin TE, Lyon LL. Rate of seeding with biopsies and ablations of hepatocellular carcinoma: a retrospective cohort study. Hepatol Commun. 2017;1:841-51.

29. Letouze E, Shinde J, Renault V, Couchy G, Blanc JF, Tubacher E, et al. Mutational signatures reveal the dynamic interplay of risk factors and cellular processes during liver tumorigenesis. Nat Commun. 2017;8:1315.

30. Schulze K, Imbeaud S, Letouze E, Alexandrov LB, Calderaro J, Rebouissou S, et al. Exome sequencing of hepatocellular carcinomas identifies new mutational signatures and potential therapeutic targets. Nat Genet. 2015;47:505-11.

31. Nguyen PHD, Ma S, Phua CZJ, Kaya NA, Lai HLH, Lim CJ, et al. Intratumoural immune heterogeneity as a hallmark of tumour evolution and progression in hepatocellular carcinoma. Nat Commun. 2021;12:227.

32. Guichard C, Amaddeo G, Imbeaud S, Ladeiro Y, Pelletier L, Maad IB, et al. Integrated analysis of somatic mutations and focal copy-number changes identifies key genes and pathways in hepatocellular carcinoma. Nat Genet. 2012; 44:694-8. 
33. Cancer Genome Atlas Research Network. Comprehensive and integrative genomic characterization of hepatocellular carcinoma. Cell. 2017;169:1327-41.e23.

34. Zhai W, Lim TK, Zhang T, Phang ST, Tiang Z, Guan P, et al. The spatial organization of intra-tumour heterogeneity and evolutionary trajectories of metastases in hepatocellular carcinoma. Nat Commun. 2017;8:4565.

\section{AUTHOR CONTRIBUTIONS}

Conception and study design: $\mathrm{AC}$ and TM; data collection: all; data analysis: TM and $A C$; writing original draft: $T M$ and $A C$; editing and final approval: all.

\section{ETHICS APPROVAL AND CONSENT TO PARTICIPATE}

This study was classified as a national audit and clinical evaluation by the Caldecott Guardian, the primary aim of which was to evaluate the feasibility, safety and outcome of diagnostic liver biopsy. As such individual patient consent was not required.

\section{COMPETING INTERESTS}

TM has served as a consultant for Eisai, Ipsen, Astra Zeneca, Roche, Boston Scientific and Bayer. The other authors declare no competing interests.

\section{FUNDING INFORMATION}

TM was partly funded by the NIHR UCLH Biomedical Research Centre and Cancer Research UK (CR UK) Accelerator award C9380/A26813.

\section{ADDITIONAL INFORMATION}

Supplementary information The online version contains supplementary material available at https://doi.org/10.1038/s41416-021-01535-2.

Correspondence and requests for materials should be addressed to T.M.

Reprints and permission information is available at http://www.nature.com/ reprints

Publisher's note Springer Nature remains neutral with regard to jurisdictional claims in published maps and institutional affiliations. Attribution 4.0 International License, which permits use, sharing, adaptation, distribution and reproduction in any medium or format, as long as you give appropriate credit to the original author(s) and the source, provide a link to the Creative Commons license, and indicate if changes were made. The images or other third party material in this article are included in the article's Creative Commons license, unless indicated otherwise in a credit line to the material. If material is not included in the article's Creative Commons license and your intended use is not permitted by statutory regulation or exceeds the permitted use, you will need to obtain permission directly from the copyright holder. To view a copy of this license, visit http://creativecommons. org/licenses/by/4.0/.

(c) The Author(s) 2021 